\title{
Research on Siding Mode Controller of High-Speed Maglev Train Under Aerodynamic Load
}

Meng-juan Liu

Chinese Academy of Sciences Institute of Mechanics

Han Wu ( $\nabla$ wuhan@imech.ac.cn )

Chinese Academy of Sciences Institute of Mechanics

Xiao-Hui Zeng

Chinese Academy of Sciences Institute of Mechanics

Bo Yin

Chinese Academy of Sciences Institute of Mechanics

Zhan-zhou Hao

Chinese Academy of Sciences Institute of Mechanics

Original Article

Keywords: High speed maglev train, Aerodynamic load, Siding mode controller, Primary suspension, Electromagnet fluctuation

Posted Date: November 30th, 2021

DOI: https://doi.org/10.21203/rs.3.rs-1106149/v1

License: (c) (i) This work is licensed under a Creative Commons Attribution 4.0 International License.

Read Full License 


\section{Title page}

\section{Research on siding mode controller of high-speed maglev train under aerodynamic load}

1. Meng-juan Liu

Key Laboratory for Mechanics in Fluid Solid Coupling Systems, Institute of Mechanics, Chinese Academy of Sciences, Beijing, 100190, China

School of Engineering Science, University of Chinese Academy of Sciences, Beijing, 100049, China

E-mail: liumengjuan@imech.ac.cn

2. Han Wu (Corresponding author)

Key Laboratory for Mechanics in Fluid Solid Coupling Systems, Institute of Mechanics, Chinese Academy of Sciences, Beijing, 100190, China

School of Engineering Science, University of Chinese Academy of Sciences, Beijing, 100049, China

E-mai1: wuhan@imech.ac.cn

3. Xiao-Hui Zeng

Key Laboratory for Mechanics in Fluid Solid Coupling Systems, Institute of Mechanics, Chinese Academy of Sciences, Beijing, 100190, China

School of Engineering Science, University of Chinese Academy of Sciences, Beijing, 100049, China

State Key Laboratory of Coastal and Offshore Engineering, Dalian University of Technology, Dalian, China

E-mail: zxh@imech. ac.cn

4. Bo Yin 
Key Laboratory for Mechanics in Fluid Solid Coupling Systems, Institute of Mechanics, Chinese Academy of Sciences, Beijing, 100190, China

School of Engineering Science, University of Chinese Academy of Sciences, Beijing, 100049, China

E-mai1: yinbo@imech.ac.cn

5. Zhan-zhou Hao

Key Laboratory for Mechanics in Fluid Solid Coupling Systems, Institute of Mechanics, Chinese Academy of Sciences, Beijing, 100190, China

School of Engineering Science, University of Chinese Academy of Sciences, Beijing, 100049, China

E-mail address: haozhanzhou@imech. ac. cn 


\title{
Research on siding mode controller of high-speed maglev train under aerodynamic load
}

\author{
Meng-juan Liu ${ }^{\mathrm{a}, \mathrm{b}}$, Han Wu ${ }^{\mathrm{a}, \mathrm{b}}{ }^{*}, \mathrm{Xiao}^{-H u i}$ Zeng $^{\mathrm{a}, \mathrm{b}, \mathrm{c}}$, Bo Yin ${ }^{\mathrm{a}, \mathrm{b}}$, Zhan-zhou Hao \\ $\mathrm{a}, \mathrm{b}$
}

a Key Laboratory for Mechanics in Fluid Solid Coupling Systems, Institute of Mechanics, Chinese Academy of Sciences, Beijing, China

b School of Engineering Science, University of Chinese Academy of Sciences, Beijing, China

c State Key Laboratory of Coastal and Offshore Engineering, Dalian University of Technology, Dalian, China

\section{Abstract}

The high-speed maglev train will be subjected to extremely obvious aerodynamic load during operation, it will also be subjected to instantaneous aerodynamic impact load in the case of passing, which will bring extreme challenges to the suspension stability and safe operation of the train. It is necessary to consider the influence of aerodynamic load and shock wave in the

\footnotetext{
* Corresponding author. E-mail address: wuhan@imech. ac. cn (Han Wu)
} 
design of suspension control algorithm. Traditional proportion integration differentiation (PID) control cannot follow the change of vehicle parameters or external disturbance, which is easy to cause suspension fluctuation and instability. In order to improve the suspension stability and vibration suppression of high-speed maglev train under aerodynamic load and impact, a controller based on sliding mode technique is designed in this paper, and in this controller, the deformation of the primary suspension is introduced to replace the aerodynamic load on the electromagnet. In order to verify the control performance of the designed controller, the dynamic simulation model of train with three vehicles is established, and the dynamic response of the train under the condition of passing in open air is calculated. Compared with the PID controller, it is verified that the sliding mode control (SMC) method proposed in this paper can effectively restrain the electromagnet fluctuation of the train under aerodynamic load.

Key words: High speed maglev train; Aerodynamic load; Siding mode controller; Primary suspension; Electromagnet fluctuation

\section{Introduction}

The speed of the wheel-rail train is increasing gradually in order to meet the needs of people' s life, but the increasing of speed will be restricted by 
the limit of wheel-rail adhesion. The appearance of the maglev train has changed this situation. The maglev train relies on the electromagnetic force between the levitation electromagnet and the track to realize suspension, and uses the guidance electromagnet to achieve the guidance function. Compared with the traditional vehicle, it has the advantages of low energy consumption, low environmental impact, low noise, less maintenance and strong climbing ability. In recent years, maglev train has made significant progress, and China's maglev system at $600 \mathrm{~km} / \mathrm{h}$ has been launched on July 20, 2021. Since the aerodynamic load is directly proportional to the square of the speed, the aerodynamic load must increase sharply with the increase of the vehicle speed. Taking the of Shanghai maglev demonstration line as an example, the numerical calculation shows that if the train runs at $600 \mathrm{~km} / \mathrm{h}$, the average aerodynamic lift of the tail car can reach 10 tons, and the instantaneous lift can reach 14 tons when the train passing another train. The stable suspension of EMS high speed maglev vehicle is realized by controlled vertical electromagnetic force, such a large aerodynamic lift and impact will have momentous influence on the suspension stability and safety of the train. Kwon et al. [1] performed numerical simulation on the response of maglev vehicle passing through the suspension bridge and was subjected to gusts of wind. The research shows that the ride comfort of the maglev vehicle is reduced due to the low frequency vibration of the vehicle caused by the bridge and turbulence wind. Yau [2] considered the aerodynamic load caused 
by unstable air flow and calculated the response of the vehicle-guideway coupling system. The results show that the aerodynamic load may cause the significant acceleration amplification of the high-speed maglev vehicle. Wu and Shi [3] completed the numerical analysis of dynamic response of maglev vehicle body under wind field. Simulation of the lateral vibration response of maglev vehicle when it passing in open air has been completed by some Liu and Tian [4]. Takizawa [5] studied the comfort of the MLX01 Maglev train when it passing at $500 \mathrm{~km} / \mathrm{h}$.

For EMS maglev train, due to the inherent instability of electromagnetic levitation, it is necessary to exert control on the levitation system to maintain stable operation, therefore, the response of EMS maglev train under external disturbance is closely related to the control algorithm. At present, the control algorithm of high-speed maglev train is still based on PID control, which realizes feedback control by calculating the acceleration of electromagnet and the difference between actual gap and rated gap. However, PID control does not involve the parameters of vehicle system and external disturbance. When the train is subjected to the aerodynamic load and impact, the control algorithm does not change with it, which is easy to cause the fluctuation and instability of suspension gap. Common state feedback control is difficult to meet the control requirements, so algorithms such as neural network control, genetic algorithm control and sliding mode control have been used to magnetic levitation system. Among them, sliding mode control is gradually carried into the control of 
magnetic levitation system because of its strong robustness and anti-disturbance. In recent years, many scholars have applied sliding mode control technology to the levitation control of maglev train.

Molero et al. [6] used the sliding mode control to the magnetic levitation ball to control the position of the nonlinear system composed of the iron ball and the electromagnetic coil. Wang [7] analyzed a single-axis magnetic levitation system and then designed an adaptive sliding mode control which could deal with unknown parameters to complete the guidance and positioning of the system. Bandal et al. [8] designed a sliding mode controller for position control of electromagnet, which based on the concept of proportional integral switching surface, and compared it with the feedback linearization controller. Yang et a1. [9] developed a new dynamic sliding surface by combining the disturbance estimation value and its high-order derivative information, and proposed a continuous dynamic sliding mode control (CDSMC) method that can be used in suspension control system. Benomair et al. [10] proposed a fuzzy sliding mode controller (FSMC) which can estimate the unmeasured state using a nonlinear observer. In order to study the influence of air resistance on the dynamic response of maglev train, Gao et al. [11] proposed an operation control method based on sliding mode periodic adaptive learning control (SM-PALC) to reduce the position error and improve the robustness of the control system. Dourla et al. [12] designed a dynamic sliding mode controller by controlling the current 
through the electromagnetic coil. Chen et al. [13] introduced sliding mode control into the research of flexible track of maglev train, and designed the sliding mode adaptive state feedback controller of maglev system, then compared with PID controller, it is found that the controller can guarantee faster dynamic response and stronger robustness when considering flexible orbit disturbance. A hybrid flux density observer based on current and voltage feedback is proposed by $\mathrm{Xu}$ et al. [14], and an adaptive sliding mode controller is designed to reduce the parameter uncertainty and disturbance upper bound of the sliding mode controller.

Sliding mode control has the characteristic of variable structure, it can force the system to move according to the state trajectory of the predetermined "sliding mode" in the dynamic process according to the current state of the system, to overcome the uncertainty of the system. Because the sliding mode can be designed and is independent of parameters and disturbances, it has the advantages of fast response, insensitivity to parameter changes and disturbances, no need for on-line identification of the system and simple physical implementation. In order to overcome the influence of the aerodynamic load and suppress the vibration of the electromagnet during the running of the train, the sliding mode control technology is adopted in this paper to design the suspension controller of the maglev train. However, due to the fact that the aerodynamic loads on maglev trains cannot be measured and obtained in real 
time during the operation of trains, how to introduce the influence of aerodynamic disturbances conveniently and effectively, so that the sliding mode controller can change its structure in time when it is subjected to aerodynamic load and impact, no scholar has given a solution at present.

Given that the vibration caused by the aerodynamic load and impact acting on the maglev train body will be transmitted to the electromagnet through the secondary suspension, the maglev frame and the primary suspension, so the fluctuation of primary suspension force can be regarded as the disturbance of aerodynamic load to the electromagnetic suspension system. Therefore, this paper considers the influence of primary suspension force disturbance on a single point suspension system, and derives a sliding mode controller considering the influence of primary suspension deformation disturbance. Based on TR08 maglev train, the dynamic simulation model of high-speed maglev train with three vehicles is presented. Then, the sliding mode controller is applied to the maglev train, and the dynamic response of the high-speed maglev train passing in open air is calculated, the control results are compared with those of PID controller and sliding mode controller without considering the primary suspension distortion disturbance. The analysis shows that compared with PID controller, sliding mode controller can perform better in restraining the vibration response of the maglev train under aerodynamic load. Moreover, considering the primary suspension deformation disturbance in the sliding mode controller, the load on 
the train can be reflected into the control process, so that the suspension gap of the train can be controlled and adjusted more effectively.

\section{Single point suspension model}

A single point two-stage suspension model was established in this paper, as shown in Fig. 1. A sliding mode controller can be presented on the basis of this model.

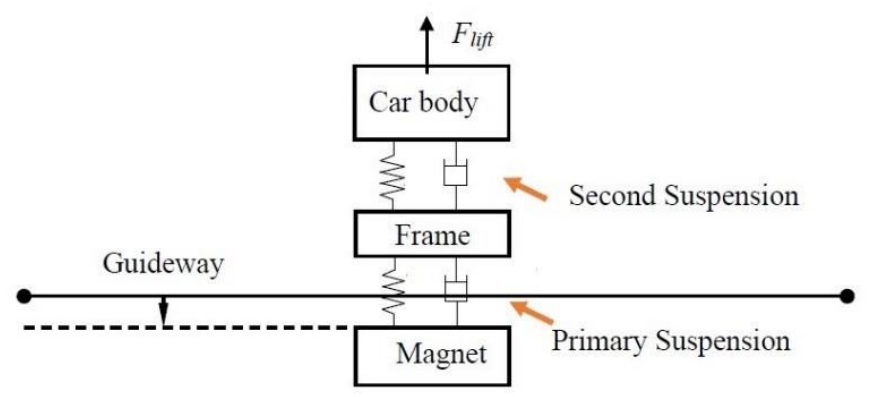

Fig. 1 Schematic diagram of single point two-stage suspension system

The vertical motion equation of the electromagnet can be written as:

$$
m \ddot{z}=m g-F(i, z)+f_{s t}(t)+f_{u n s t}(t)
$$

The levitation force between the electromagnet and the guide rail is calculated by the classical electromagnetic force formula as follows:

$$
F(i, z)=\frac{\mu_{0} A N^{2} I^{2}}{4 z^{2}}
$$


In Eq. (2), $\mu_{0}$ is the air permeability, $z$ is the suspension gap, $A$ is the effective area of the electromagnet, $N$ is the turns of the coil, $f_{s t}(t)$ and $f_{\text {unst }}$ ( $t$ ) represent the balance value and the fluctuation of primary suspension force, respectively.

The maglev train directly adjusts the current in the electromagnet through the suspension control unit. The dynamic equation can be written as follows:

$$
\ddot{z}=-\frac{\mu_{0} A N^{2}}{4 m}\left[\frac{I(t)}{z(t)}\right]^{2}+g+\frac{f_{s t}(t)+f_{u n s t}(t)}{m}
$$

Let $x_{1}=z(t), x_{2}=\dot{z}(t)$, the state equation of the electromagnet levitation system can be written as follows:

$$
\left\{\begin{array}{c}
\dot{x}_{1}(t)=x_{2}(t) \\
\dot{x}_{2}(t)=-\frac{\mu_{0} A N^{2}}{4 m}\left[\frac{I(t)}{x_{1}(t)}\right]^{2}+g+\frac{f_{s t}(t)+f_{u n s t}(t)}{m}
\end{array}\right.
$$

Set $\left[\frac{I(t)}{x_{1}(t)}\right]^{2}$ as $u$ to design the suspension controller, and rewrite the dynamic equation as:

$$
\ddot{z}=-\frac{\mu_{0} A N^{2}}{4 m} u+g+\frac{f_{s t}(t)+f_{u n s t}(t)}{m}
$$

The state equation is rewritten as:

$$
\left\{\begin{array}{c}
\dot{x}_{1}(t)=x_{2}(t) \\
\dot{x}_{2}(t)=-\frac{\mu_{0} A N^{2}}{4 m} u+g+\frac{f_{s t}(t)+f_{u n s t}(t)}{m}
\end{array}\right.
$$

or

$$
\dot{\boldsymbol{X}}=\boldsymbol{A} \boldsymbol{X}+\boldsymbol{B}\left(-\frac{\mu_{0} A N^{2}}{4} u+m g+f_{s t}(t)+f_{\text {unst }}(t)\right)
$$


Where, $\boldsymbol{A}=\left[\begin{array}{ll}0 & 1 \\ 0 & 0\end{array}\right], \boldsymbol{X}=\left[\begin{array}{ll}x_{1} & x_{2}\end{array}\right]^{T}, \boldsymbol{B}=\left[\begin{array}{ll}0 & \frac{1}{m}\end{array}\right]^{T}$.

\section{Design of sliding mode controller}

\section{(1) Design of sliding surface}

The switching function $\mathrm{s}(\mathrm{x})$ is designed and the switching surface $\mathrm{s}(\mathrm{x})=0$ is determined to ensure the asymptotic stability and good dynamic characteristics of the dynamic system on the sliding surface.

Define tracking error

$$
\boldsymbol{E}=\left[\begin{array}{c}
e \\
\dot{e}
\end{array}\right]=\left[\begin{array}{l}
z-z_{0} \\
\dot{z}-\dot{z}_{0}
\end{array}\right]=\left[\begin{array}{l}
x_{1}-z_{0} \\
x_{2}-\dot{z}_{0}
\end{array}\right]
$$

$z_{0}, \dot{z}_{0}$ are the irregularity of the track and its derivative, respectively

The switching function of the system is designed as:

$$
s(x)=\boldsymbol{C E}=c e+c_{d} \dot{e}=c\left(x_{1}-z_{0}\right)+c_{d}\left(x_{2}-\dot{z}_{0}\right)
$$

In order to guarantee the asymptotic stability on the sliding surface of the system, that is $s(x)=0$, it is necessary to meet $c>0$.

(2) Design of sliding mode law

The design of sliding mode control law should meet the following requirements: it can make the system state enter the sliding mode state from 
any initial point, and keep it on the sliding mode surface stably and reliably. The law of convergence mainly has: constant rate of convergence, exponential rate of convergence and power rate of convergence. The exponential reaching law can effectively reduce buffeting vibration in the form of:

$$
\dot{s}=-\xi \operatorname{sgn}(s)-k s, \xi>0, k>0
$$

According to Eq. (9), we can get:

$$
\dot{s}=c\left(\dot{x}_{1}-\dot{z}_{0}\right)+\left(c_{d} \dot{x}_{2}-\ddot{z}_{0}\right)
$$

According to Eq. (6), Eq. (10) and Eq. (11), the control law of the system is:

$$
u=-\frac{4 m}{\mu_{0} A N^{2}}\left\{[-\xi \operatorname{sgn}(s)-k s]-c\left[c_{d} x_{2}(t)-\dot{z}_{0}\right]-\left(g+\frac{f_{s t}(t)+f_{u n s t}(t)}{m}-\ddot{z}_{0}\right)\right\}
$$

In Eq. (12), $c_{d}=1 ; c, \xi, k$ are undetermined constants. The electromagnet is expected to levitate stably at $10 \mathrm{~mm}, z_{0}=10 \mathrm{~mm}, \quad \dot{z}_{0}=\ddot{z}_{0}=0$.

Because moving point of the system still has velocity when it reaches the switching surface, and it will continue to pass through the switching surface under the action of inertia, the moving point in the sliding mode will be accompanied by high frequency vibration, thus forming buffeting vibration. In the exponential approach law, $\xi \operatorname{sgn}(s)$ is an equal speed convergence term, when $S$ approaches 0 , the approach speed is $\xi$ instead of 0 , which can be guaranteed to reach the sliding surface in finite time. The second term is exponential term. 
When $S$ is large, the system state can converge to 0 at a large speed. In the exponential approach, the approach velocity approaches 0 from a larger velocity, which not only shortens the approach time, but also decreases the velocity when the moving point reaches the switching surface. In order to further suppress the buffeting vibration of sliding mode control, the saturation function is used to replace the switching function, and the control law is changed as follows:

$$
\dot{s}=-\xi \cdot \operatorname{sat}\left(\frac{s}{\mu}\right)-k \cdot s=\mathrm{slaw}, \xi>0, k>0, \mu>0
$$

Where sat is a saturation function whose expression is:

$$
\operatorname{sat}\left(\frac{s}{\mu}\right)=\left\{\begin{array}{cc}
\frac{s}{\mu} & \left|\frac{s}{\mu}\right| \leq 1 \\
\operatorname{sgn}\left(\frac{s}{\mu}\right) & \left|\frac{s}{\mu}\right|>1
\end{array}\right.
$$

The control law of the system can ultimately be determined as:

$$
u=-\frac{4 m}{\mu_{0} A N^{2}}\left\{\left[-\xi \cdot \operatorname{sat}\left(\frac{s}{\mu}\right)-k s\right]-c\left[x_{2}(t)-\dot{z}_{0}\right]-\left(g+\frac{f_{s t}(t)+f_{\text {unst }}(t)}{m}-\ddot{z}_{0}\right)\right\}
$$

Considering that the vibration caused by aerodynamic load and impact acting on the maglev train body will be transmitted to the electromagnet through the secondary suspension, maglev frame and primary suspension, therefore the fluctuation of primary suspension force can be regarded as the disturbance of aerodynamic load or vehicle body vibration to the electromagnetic suspension system. In the sliding mode control law (Eq. (15)), the variation term $f_{\text {unst }}$ of 
the primary suspension load is included. In this way, the variable structure characteristics of the sliding mode controller can be well reflected, in other words, the controller can continuously change according to the aerodynamic load disturbance of the vehicle, and force the suspension system to move according to the state trajectory of the predetermined "sliding mode", so as to overcome the uncertainty of the maglev train.

In order to adjust the parameters of the controller, the variation of the primary suspension force is expressed by Eq. (16), which $\Delta Z_{\text {primary }}$ is the deformation of primary suspension spring and $K_{p r i}$ is an adjustable parameter.

$$
f_{\text {unst }}(t)=K_{\text {pri }} \cdot \Delta Z_{\text {primary }}
$$

\section{The dynamic model of maglev train with three vehicles}

The dynamic model of single vehicle TR08 maglev train is shown in Fig. 2.

Each vehicle is mainly composed of the following parts: car body (1 piece), frame (4 pieces), pillow beam (8 pairs), pendel (8 pairs), traction pull rod (4 pieces), traction/suspension magnet (8 pairs for intermediate vehicle and 7. 5 pairs for lead vehicle), brake magnet (1 pair), guide magnet (6 pairs), air spring (8 pairs), car body transverse elastic holder or rubber spring, traction/suspension electromagnet and suspension frame unit support (16 pairs for intermediate vehicle and 15 pairs for lead vehicle), brake electromagnet support, etc. 


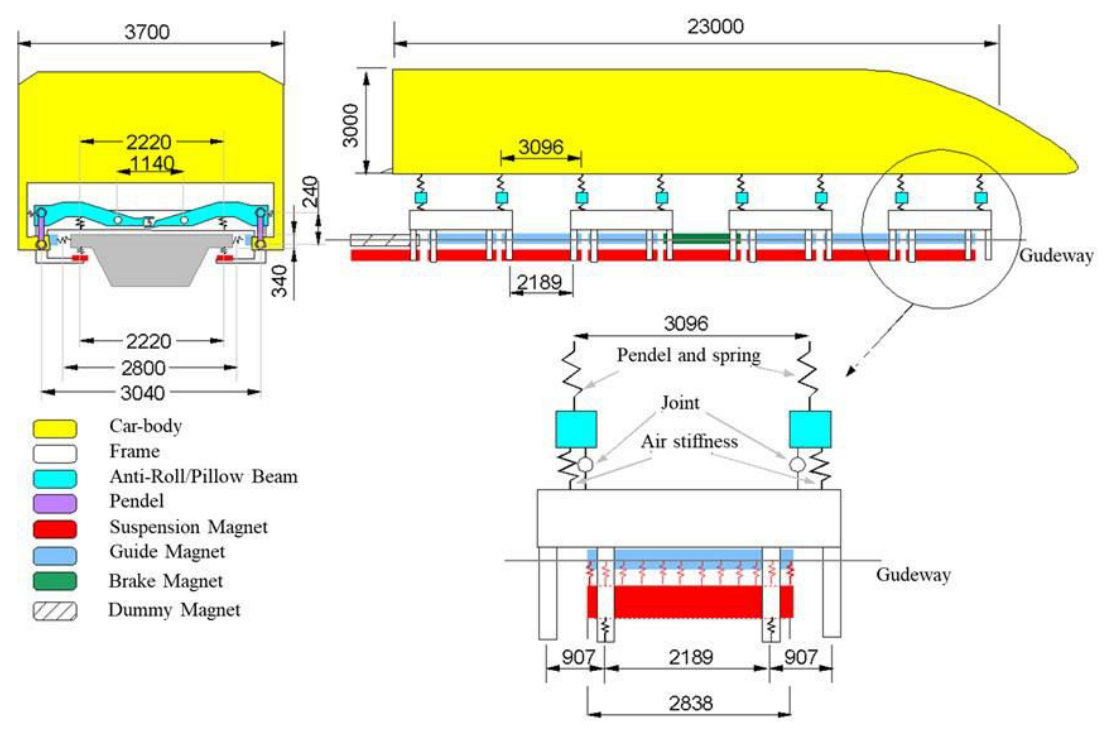

Fig. 2 Vehicle dynamics mode1

In the modeling, because the suspension frame is connected by two C-shaped frames, and the longitudinal beam connected in the middle has large flexible deformation, each suspension frame is simplified into two semi-maglev frames, which are connected through component. Other parts are considered as rigid bodies and their flexible deformation is ignored. The suspension structure of the vehicle embodies the independence of stiffness in: the vertical, transverse and longitudinal traction of the secondary system are provided by air spring, pendel and lateral additional spring/holder and traction device, respectively. The primary suspension is independently provided by supporting rubber parts or arthrosis structures of the traction electromagnet and the guide (including brakes, replacements) electromagnet. The track and vehicle are coupled by electromagnetic force, and the suspension electromagnetic force is actively controlled by the control system, so as to realize the suspension of the vehicle. 
In this paper, the dynamic simulation model of train was established by using multi-body dynamics software. Based on the dynamic model shown in Fig. 2, a simulation model of TR08 single vehicle was established, then the maglev train with three vehicles was formed, which is shown in Fig. 3, and the position of electromagnet on each train is marked in the figure.

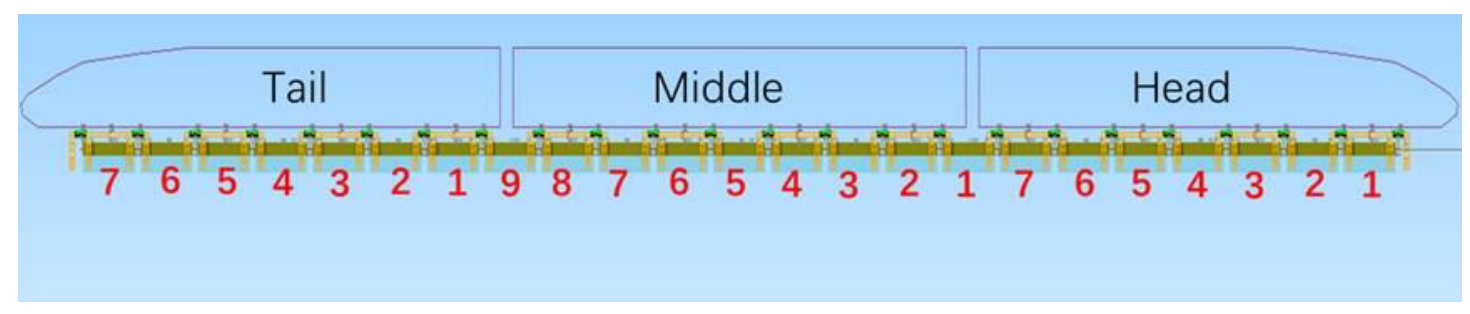

Fig. 3 Dynamic model of TR08 maglev train

In this paper, the train was controlled dispersedly, so that the design of the whole vehicle suspension control system could be completed by designing the single electromagnet suspension control system, which could make the control system more simplified. Each suspension electromagnet of TR08 train has 12 coils, six of which share one control unit, and each control unit is independent of each other. This paper used SIMULINK to simulate the control module, based on the sliding mode control technology proposed in this paper, the single channel suspension control scheme is present as follows: 


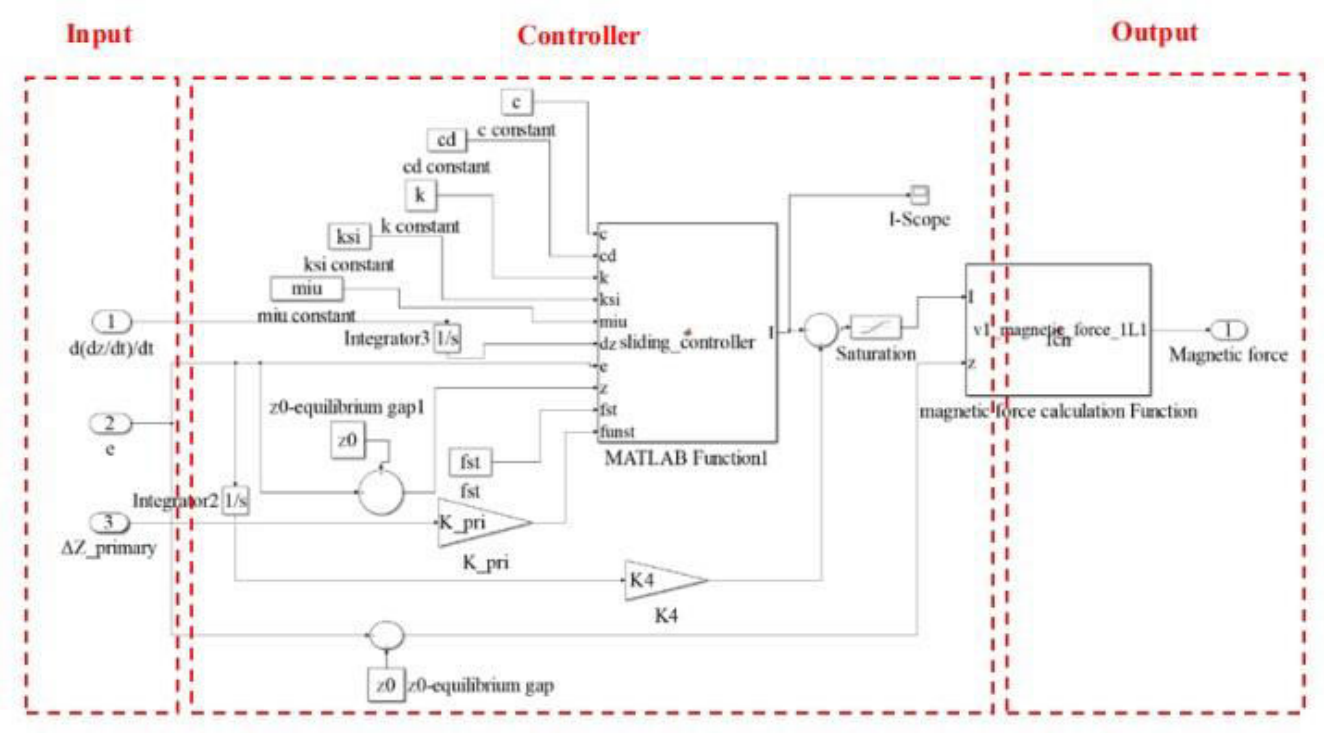

Fig. 4 Single channel SMC suspension control system model

On the basis of the dynamic simulation model and the suspension control model of the train with three vehicles, the data transmission of the multi-body dynamic model and the control model is realized through the SIMAT interface in MATLAB, so as to realize the coupling simulation between multi-body dynamics and suspension control of TR08 train. Each single channel control unit consists of input, controller and output. Each control unit receives the fluctuations of electromagnet suspension gap, acceleration and primary suspension force obtained by vehicle dynamics simulation, calculates these data to obtain the desired current value, then calculates the output electromagnetic suspension force, and finally returns it to the vehicle dynamics simulation model. 
At the same time, in order to demonstrate the control effect of the proposed sliding mode controller, this paper also used the PID control algorithm as the comparison. The single channel PID controller is shown in Fig. 5.

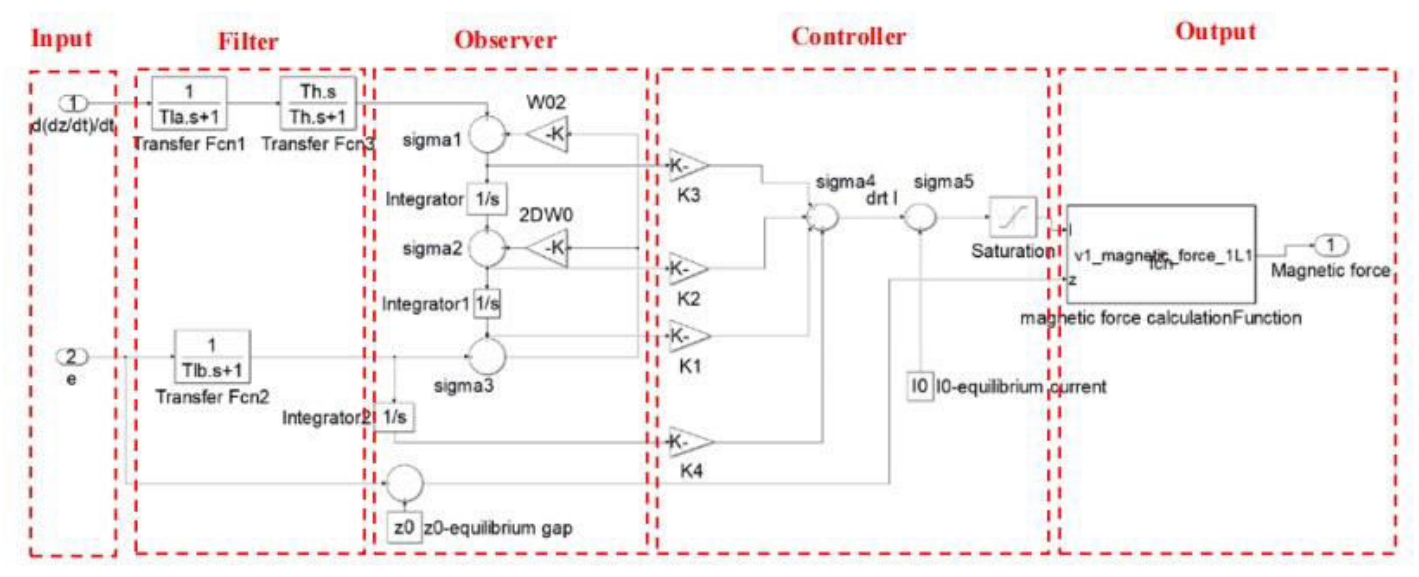

Fig. 5 Single channel PID suspension control system model

Each single channel PID control unit is composed of filter, observer and controller. The estimated value in the state observer is a weighted combination of air gap, velocity and acceleration. Each control unit receives the electromagnet suspension gap and acceleration that input from the vehicle dynamics model, then these signals are filtered and observed through the SIMULINK, and the electromagnetic levitation force is calculated by the controller and fed back to the dynamic simulation module. 


\section{Analysis of train response under aerodynamic load}

\section{(1) Aerodynamic load data}

Based on the CFD method, the aerodynamic load of the train passing in open air at $300 \mathrm{~km} / \mathrm{h}, 400 \mathrm{~km} / \mathrm{h}$ and $500 \mathrm{~km} / \mathrm{h}$ was simulated. Under different operating speeds, the variation of aerodynamic load curve acting on the train is basically the same, but with the increase of vehicle speed, the amplitude of load will also increase. The aerodynamic lateral force, lift force and pitching moment on the train when it runs at $500 \mathrm{~km} / \mathrm{h}$ are shown in Fig. 6. The aerodynamic moment is the result of taking the center of mass of car body as the moment point. The blue, green and orange solid lines in the figures represent the aerodynamic load on the head, middle and tail vehicle of the train during operation, respectively, while the range covered by the black dotted line in the figure represents the passing stage of the train in the open air. Obviously, when the train is running, the tail train receives the largest aerodynamic load, followed by the head car, the middle car is smaller. It is noted that the aerodynamic load fluctuates obviously in the train passing stage. 

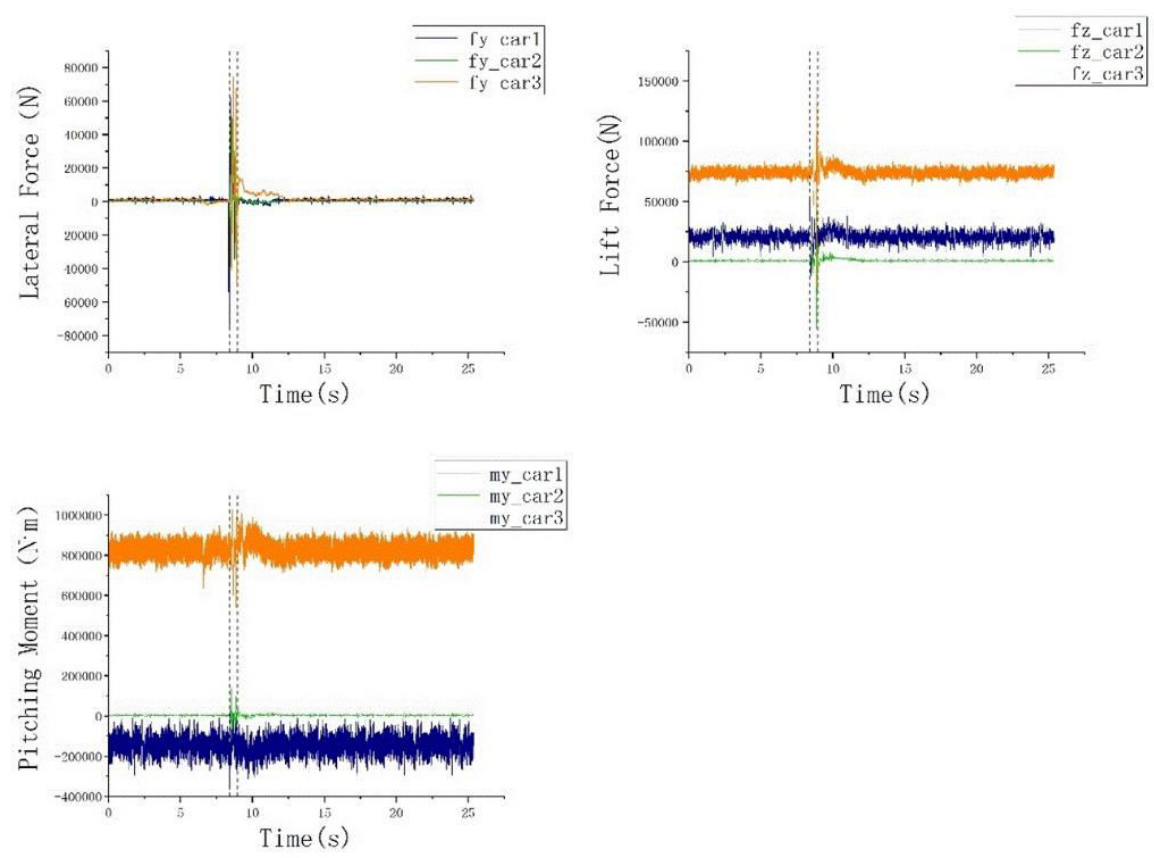

Fig. 6 Aerodynamic load at $500 \mathrm{~km} / \mathrm{h}$

This paper applied the above aerodynamic force and moment to the vehicle centroid in the dynamic model, carried out the motion simulation of maglev train under aerodynamic loads, then compared the dynamic response of the train under using PID control, traditional sliding mode control (SMC) and sliding mode control considering primary suspension disturbance (SMC_P), including the vehicle ride quality, the displacement of the train and the gap fluctuation of electromagnets. In PID control, feedback control parameters are set to: $\mathrm{K}_{1}=7000$, $K_{2}=200, K_{3}=0.5, K_{4}=1000$. The parameters of sliding mode controller are set to: $c=100, \quad \xi=20, \quad k=80, \quad K_{p r i}=-2 \times 10^{7} / 6.0$. 


\section{(2) Car body response analysis}

The results about Sperling ride index of trains running at different speeds in open air under aerodynamic loads are shown in Table 1.

Table 1 Comparison of vertical Sperling ride index

\begin{tabular}{|c|c|c|c|c|}
\hline Speed $(\mathrm{km} / \mathrm{h})$ & Car body & PID （mm） & SMC (mm) & SMC_P (mm) \\
\hline \multirow{3}{*}{300} & Head car & 1. 330317 & 1. 334163 & 1. 332673 \\
\hline & Middle car & 1. 138362 & 1. 144525 & 1. 151148 \\
\hline & Tail car & 1. 670477 & 1. 693833 & 1. 710850 \\
\hline \multirow{3}{*}{400} & Head car & 1. 479943 & 1. 506137 & 1. 513859 \\
\hline & Middle car & 1. 250410 & 1. 493161 & 1. 503598 \\
\hline & Tail car & 1.922125 & 1. 904598 & 1. 891828 \\
\hline \multirow{3}{*}{500} & Head car & 2. 289424 & 2. 317263 & 2.334752 \\
\hline & Middle car & 1. 608220 & 1. 618358 & 1. 626261 \\
\hline & Tail car & 2. 021350 & 2. 001179 & 1. 982947 \\
\hline
\end{tabular}

It is apparent that Sperling ride index of the train gradually increases with the increase of speed. In the case of PID control, traditional sliding mode (SMC) control and sliding mode with primary suspension force disturbance 
(SMC_P), the results about Sperling ride index have little difference and are all less than 2.5, which means the train has excellent stability.

Table 2 shows the heave motion amplitudes of the car body under using three controllers when it is passing in open air at different speeds. It is obvious that the vertical vibration amplitude of the head train is the smallest and the tail train is the largest under the different speeds, and the vibration amplitude of the train increases gradually with the raise of the speed. Under the three controllers, the vertical vibration amplitudes of the car body are close and the response results are excellent.

Table 2 Vertical vibration amplitudes of the car body

\begin{tabular}{|c|c|c|c|c|}
\hline Speed $(\mathrm{km} / \mathrm{h})$ & Car body & PID (m) & SMC (m) & SMC_P (m) \\
\hline \multirow{3}{*}{300} & Head car & 0.0144051 & 0.0142736 & 0.0144051 \\
\cline { 2 - 5 } & Middle car & 0.0115441 & 0.0114622 & 0.0115441 \\
\cline { 2 - 5 } & Tail car & 0.0426626 & 0.0424268 & 0.0426626 \\
\hline \multirow{3}{*}{400} & Head car & 0.0229016 & 0.023062 & 0.023111 \\
\cline { 2 - 5 } & Middle car & 0.0191232 & 0.0190864 & 0.0190421 \\
\cline { 2 - 5 } & Tail car & 0.0693106 & 0.0693116 & 0.0692293 \\
\hline
\end{tabular}




\begin{tabular}{|l|c|c|c|c|}
\hline \multirow{3}{*}{500} & Head car & 0.0583298 & 0.0585040 & 0.0585755 \\
\cline { 2 - 5 } & Middle car & 0.0310934 & 0.0312598 & 0.0313184 \\
\cline { 2 - 5 } & Tail car & 0.0951066 & 0.0945006 & 0.0939576 \\
\hline
\end{tabular}

\section{(3) Levitation gap response of electromagnet}

Fig. 3 shows the distribution of electromagnets in the train. Fig. 7 - Fig.

9 show the fluctuation curves of the electromagnet levitation gap on the right at positions H1, M1, M9 and T7 when the train is running at different speeds. The black dotted line corresponds to the time when the two train meet and separate.

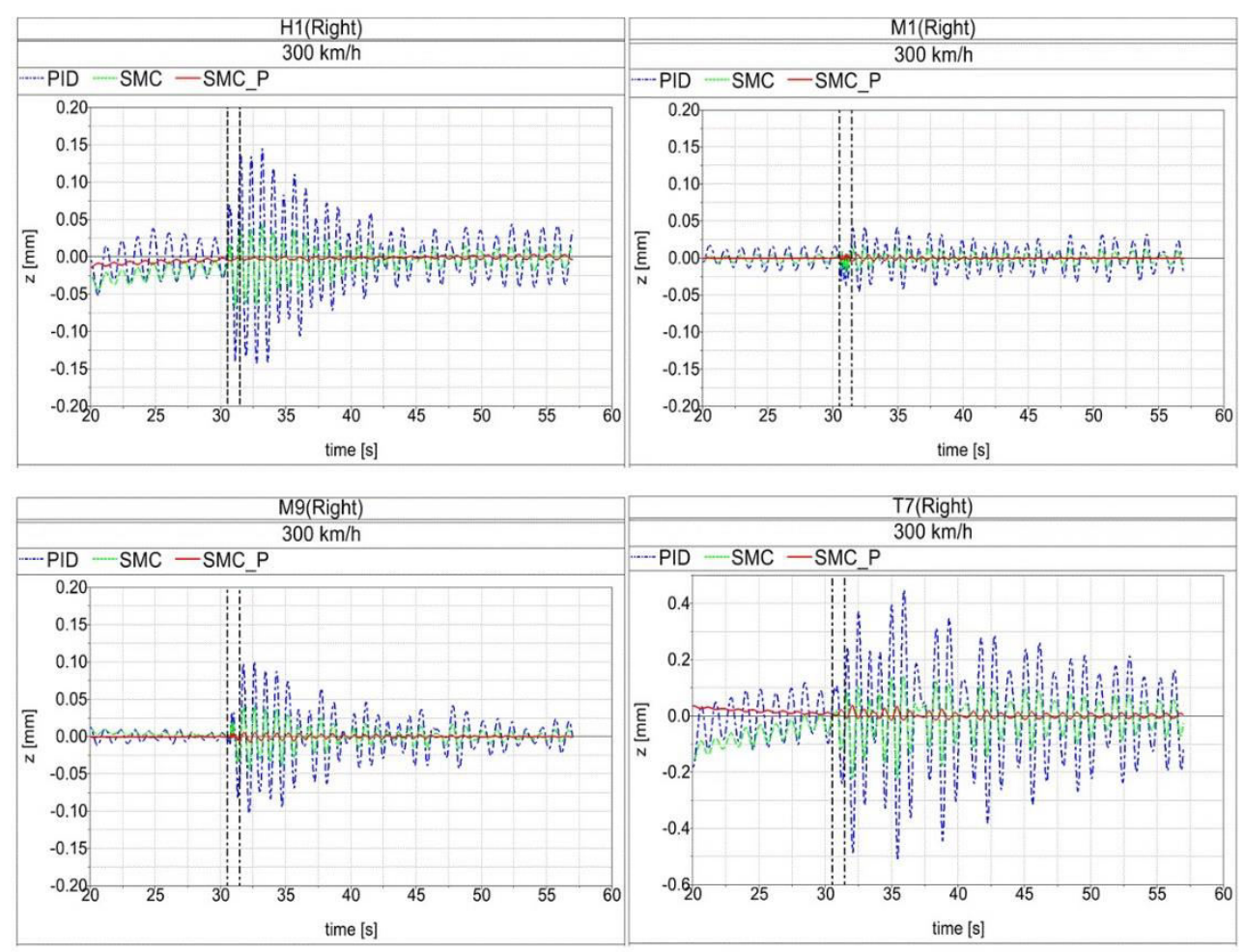


Fig. 7 Electromagnet gap fluctuation at $300 \mathrm{~km} / \mathrm{h}$ (Negative ordinate represents upward movement of electromagnet)
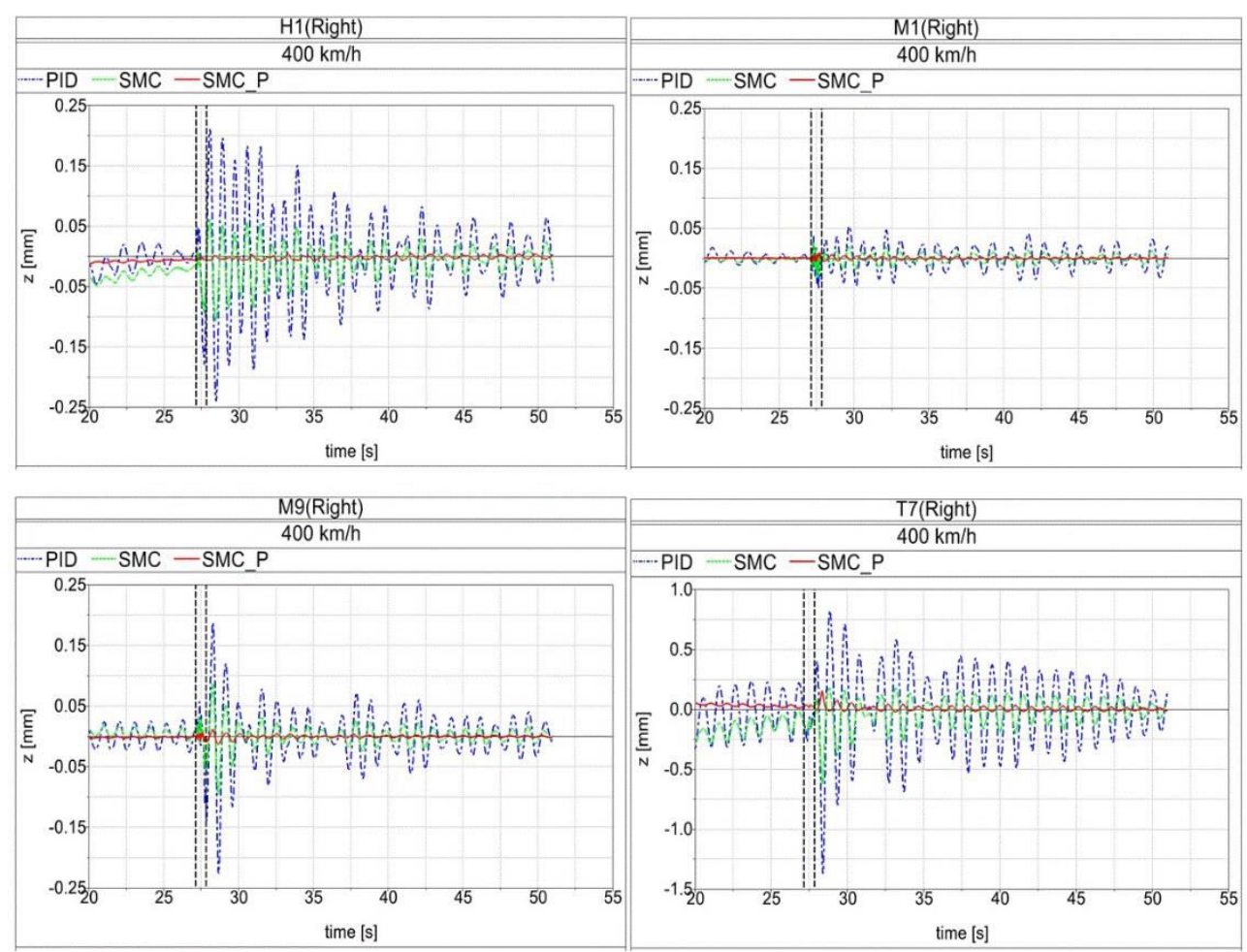

Fig. 8 Electromagnet gap fluctuation at $400 \mathrm{~km} / \mathrm{h}$ 

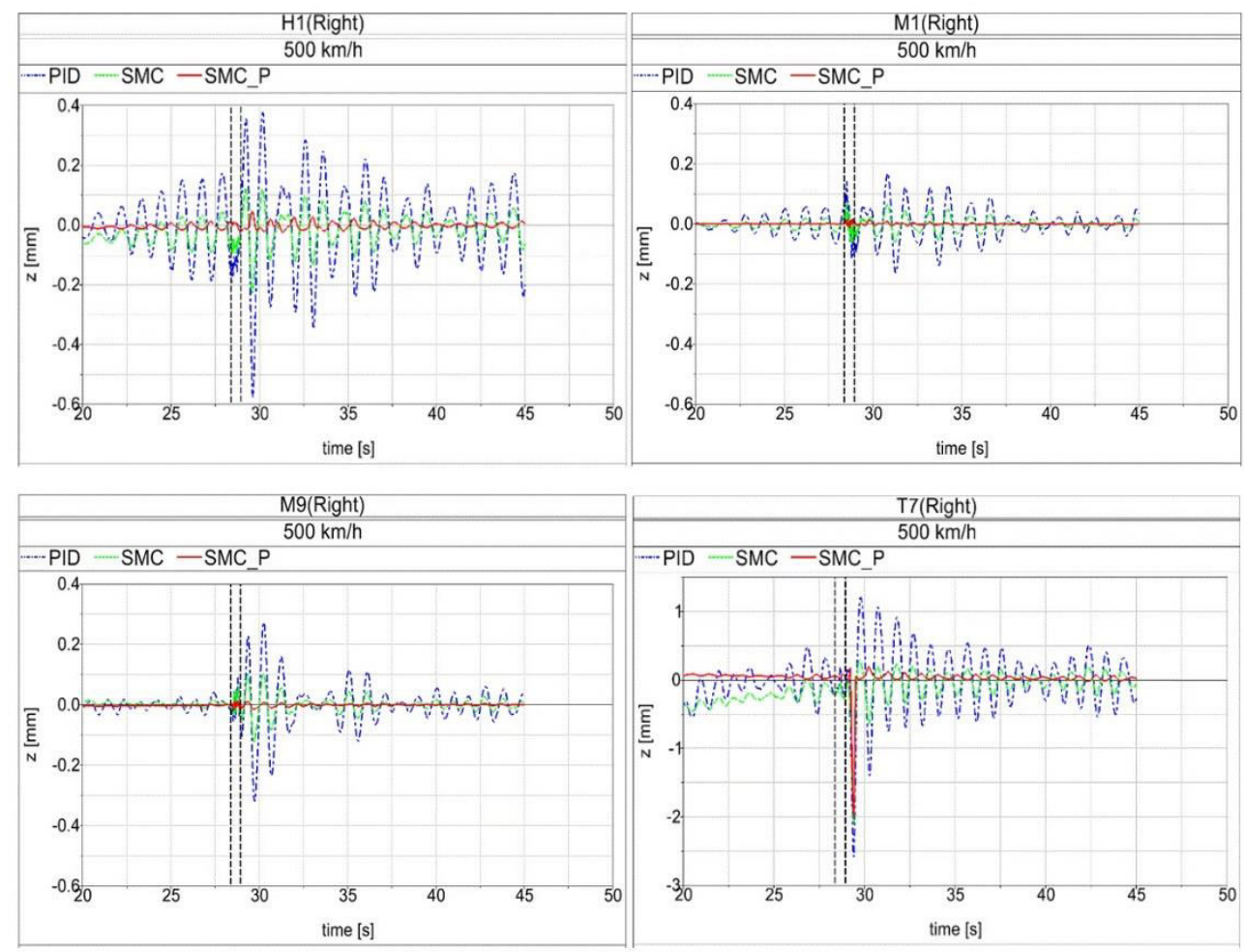

Fig. 9 Electromagnet gap fluctuation at $500 \mathrm{~km} / \mathrm{h}$

Obviously, using the sliding mode controller considering the primary suspension deformation disturbance designed in this paper, the gap fluctuation values of electromagnet at the head, middle and tail vehicle are smaller than those when using the traditional sliding mode control. Compared with using traditional PID controller, it has more excellent performance and good suspension stability.

Table 3 shows the average values of the electromagnetic levitation gap amplitude of the head car, middle car and tail car when the train passing in open air at different speeds. It is obvious compared with using traditional PID control and traditional sliding mode control, the fluctuation amplitude of 
suspension gap of the train using sliding mode control considering the disturbance of the primary force is greatly reduced at different speeds.

Table 3 Average amplitudes of electromagnet suspension gap

\begin{tabular}{|c|c|c|c|c|}
\hline Speed $(\mathrm{km} / \mathrm{h})$ & Car body & PID (mm) & SMC (mm) & SMC_P (mm) \\
\hline \multirow{3}{*}{300} & Head car & 0.12979 & 0.05320 & 0.00493 \\
\hline & Middle car & 0.05218 & 0.02080 & 0.00174 \\
\hline & Tail car & 0.21061 & 0.08984 & 0.00987 \\
\hline \multirow{3}{*}{400} & Head car & 0.17834 & 0.07774 & 0.00503 \\
\hline & Middle car & 0.06586 & 0.03113 & 0.00230 \\
\hline & Tail car & 0.41817 & 0.19068 & 0.03071 \\
\hline \multirow{3}{*}{500} & Head car & 0.31736 & 0.13087 & 0.01377 \\
\hline & Middle car & 0.11213 & 0.05458 & 0.003518 \\
\hline & Tail car & 0.62486 & 0.42089 & 0.29613 \\
\hline
\end{tabular}

In order to more specifically illustrate the fluctuation of the levitation gap of electromagnet under using three kinds of controller, Fig. 10 - Fig. 12show the levitation gap amplitudes, including all electromagnets of the whole vehicle. It can be seen that under the action of the sliding mode controller 
designed in this paper, the suspension gap amplitude of the whole train at 300 $\mathrm{km} / \mathrm{h}$ is 1 ess than $0.1 \mathrm{~mm}$, the suspension gap amplitude at $400 \mathrm{~km} / \mathrm{h}$ is 1 ess than $0.2 \mathrm{~mm}$, and the suspension gap amplitude at $500 \mathrm{~km} / \mathrm{h}$ is 1 ess than $2 \mathrm{~mm}$, showing good suspension stability.
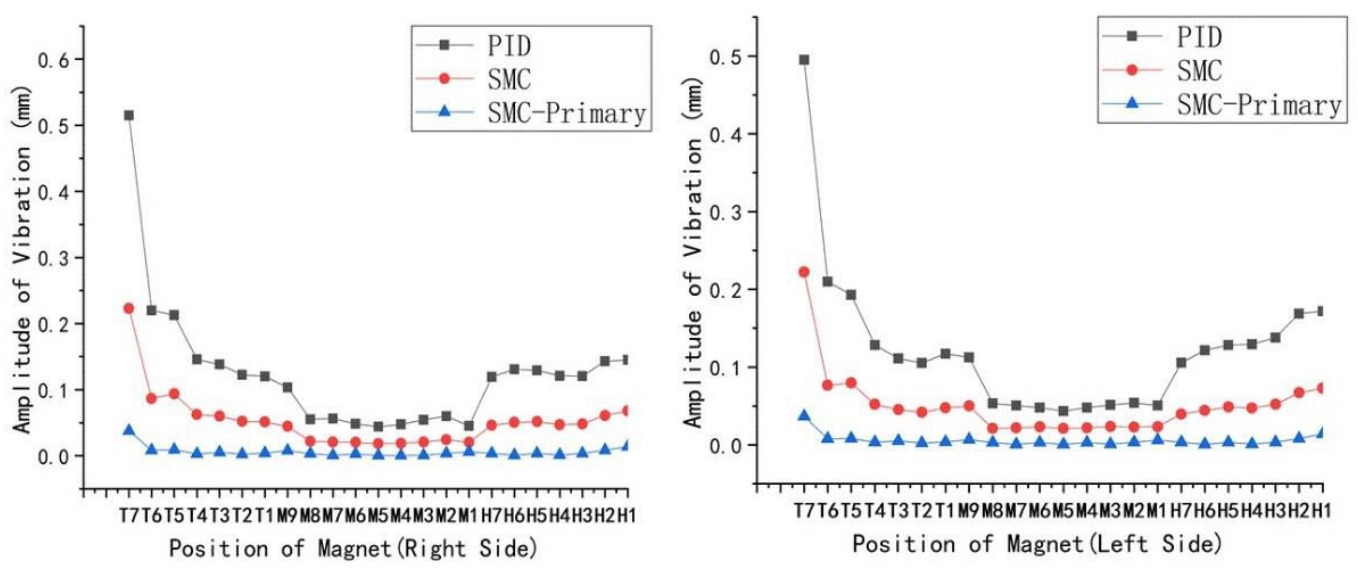

Fig. 10 Amplitude distribution of suspension gap at $300 \mathrm{~km} / \mathrm{h}$
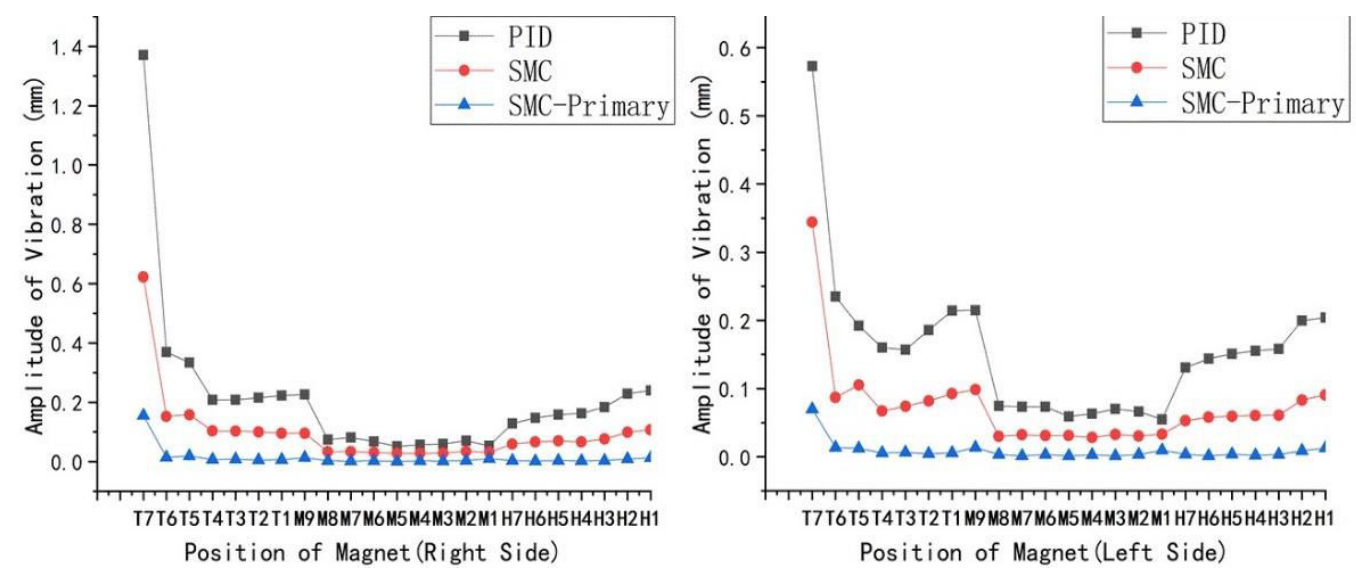

Fig. 11 Amplitude distribution of suspension gap at $400 \mathrm{~km} / \mathrm{h}$ 

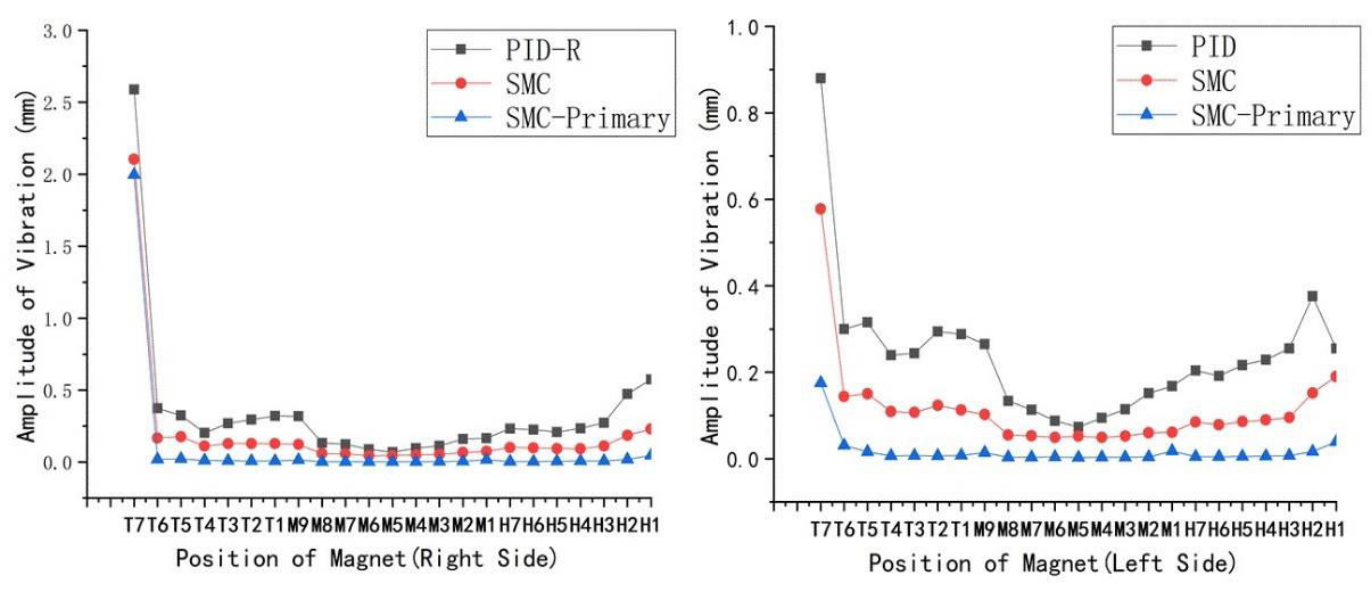

Fig. 12 Amplitude distribution of suspension gap at $500 \mathrm{~km} / \mathrm{h}$

Take the suspension gap on the right side of the train at $300 \mathrm{~km} / \mathrm{h}$ as an example: at the position $\mathrm{H} 1$ of the first electromagnet of the head train, the amplitude of the suspension gap is $0.14505 \mathrm{~mm}$ when PID control is used; The amplitude at $\mathrm{H} 1$ is $0.06786 \mathrm{~mm}$ under using the traditional sliding mode control; The amplitude at $\mathrm{H} 1$ is $0.01384 \mathrm{~mm}$ when the sliding mode control designed in this paper is used, which is reduced by $90.49 \%$ compared with PID control and $79.61 \%$ compared with using traditional sliding mode controller.

At the position $\mathrm{T} 7$ of the last electromagnet of the tail car, the amplitude of the suspension gap is $0.51499 \mathrm{~mm}$ under using PID control, and $0.22301 \mathrm{~mm}$ when using the traditional sliding mode control. The amplitude of the suspension gap at T7 is $0.03761 \mathrm{~mm}$ under using the control proposed in this paper, which is 92. 70\% 1ess than that of PID control and $83.14 \%$ less than that of the traditional sliding mode controller. 


\section{Conclusion}

In this paper, the influence of aerodynamic load on electromagnet is replaced by the disturbance of primary suspension force, then a sliding mode controller considering the disturbance of primary suspension force is designed. The dynamic simulation model of high-speed maglev train with three vehicles is established, and the dynamic response of the train passing with another train in open air is calculated. It is proved that under the action of the controller designed in this paper, the dynamic responses of train body displacement, Sperling ride index and suspension gap fluctuation meet the requirements of train operation. Then, by comparing with PID and traditional sliding mode controller, it is found that under the sliding mode control considering the disturbance of primary suspension force, the electromagnet fluctuation amplitude at each position of the train is significantly reduced, which proves that the controller proposed in this paper can effectively suppress the electromagnet fluctuation under aerodynamic load. This study can provide reference for the suspension control optimization of maglev train under aerodynamic conditions.

\section{Declaration}




\section{Availability of data and materials}

Not applicable.

\section{Competing interests}

The Authors declare that there is no conflict of interest.

\section{Funding}

This project was supported by the National Key R\&D Program of China (Grant 2016YFB1200602), the National Natural Science Foundation of China (Grants 51805522, 11672306 and 51490673), the open fund of the State Key Laboratory of coastal and offshore engineering, Dalian University of Technology (Grant LP21V1).

\section{Authors' contributions}

Mengjuan Liu: Data curation, Writing- Original draft preparation, Software,

Formal analysis.

Han Wu: Conceptualization, Methodology, Funding acquisition, Article check, Supervision.

Xiaohui Zeng: Funding acquisition, Supervision.

Bo Yin: Improve data.

Zhanzhou Hao: Improve data. 


\section{Acknowledgements}

Not applicable.

\section{Reference}

[1] Kwon SD, Lee JS, Moon JW, et al. (2008). Dynamic interaction analysis of urban transit maglev vehicle and guideway suspension bridge subjected to gusty wind. Engineering Structures, 30(12), 3445-3456, D0I:

10. 1016/ j. engstruct. 2008. 05. 003.

[2] Yau JD. (2010). Aerodynamic vibrations of a maglev vehicle running on flexible guideways under oncoming wind actions. Journal of Sound and Vibration, 329(10), 1743-1759, DOI: 10.1016/j. jsv. 2009.11. 039.

[3] Wu JJ and Shi XH. (2009). Numerical analyses of dynamic stability of maglev vehicles in crosswind field. Journal of Lanzhou University: Natural Science Edition, 45(2), 96-102.

[4] Liu TH and Tian HQ. (2005). Transverse vibration analysis of two maglev trains passing by in open air. Journal of transportation engineering, 5(1), $39-44$.

[5] Takizawa H. (2004). The vehicle dynamics characteristics of a magnetic levitation train passing another train running in opposite direction. Foreign railway vehicles, 41(1), 30-33. 
[6] Molero R, Roca J, Separovich D, et al. (2008). Nonlinear Control of an Electromagnetic System Based on Exact Linearization and Sliding Mode Control, Mechanics Based Design of Structures and Machines, 36(4), 426-445, D0I:

10. $1080 / 15397730802414921$.

[7] Wang CC, Chen MY and Fu LC. (2000). Adaptive sliding mode controller design of a maglev guiding system for application in precision positioning. Proceedings of the 2000 American Control Conference. ACC (IEEE Cat. No. OoCH36334), vol. 3, 1612-1616. DOI: 10. 1109/ACC. 2000. 879473.

[8] Bandal VS and Vernekar PN. (2009). A new approach to a sliding mode controller design for a magnetic levitation system. 2009 Asia-Pacific Conference on Computational Intelligence and Industrial Applications (PACIIA), 326-329. D0I: 10. 1109/PACIIA. 2009. 5406426.

[9] Yang J, Su J, Li S, et al. (2014) High-Order Mismatched Disturbance Compensation for Motion Control Systems Via a Continuous Dynamic Sliding-Mode Approach. in IEEE Transactions on Industrial Informatics, 10(1), 604-614. D0I: 10. 1109/TII. 2013. 2279232.

[10] Benomair AM, Firdaus AR and Tokhi MO. (2016). Fuzzy sliding control with non-linear observer for magnetic levitation systems. 2016 24th Mediterranean Conference on Control and Automation (MED), 256-261. D0I:

10. 1109/MED. 2016. 7536025. 
[11] Gao Y, Wang Y, Zhang J, et al. (2020). A sliding mode periodic adaptive learning operation control method for medium-speed maglev trains. 2020 39th Chinese Control Conference (CCC), 5548-5553. D0I:

10. 23919/CCC50068. 2020. 9188741.

[12] Dourla V, Pandey S and Junghare AS. (2017). Design and analysis of dynamic sliding mode control for magnetic levitation system. 2017 th International Conference on Power, Control \& Embedded Systems (ICPCES), 1-6. DOI : 10. 1109/ICPCES. 2017. 8117661.

[13] Chen C, Xu J, Ji W, et al. (2019). Sliding Mode Robust Adaptive Control of Maglev Vehicle’ s Nonlinear Suspension System Based on Flexible Track: Design and Experiment. in IEEE Access, vol. 7, 41874-41884. D0I:

10. 1109/ACCESS. 2019. 2906245.

[14] Xu J, Sun Y, Gao D, et al. (2018). Dynamic Modeling and Adaptive Sliding Mode Control for a Maglev Train System Based on a Magnetic Flux Observer. in IEEE Access, vol. 6, 31571-31579, DOI: 10. 1109/ACCESS. 2018. 2836348. 